\title{
Effect of Taekwondo Poomsae Training on Weight Loss of Obese Children
}

\begin{abstract}
Qianfeng $\operatorname{Lin}^{1, *}$
${ }^{1}$ Chonbuk National University, Chonju, Korea

*Corresponding author.

ABSTRACT

This study takes obese children as the research objects to explore the influence of Taekwondo Poomsae training on obese children. The objects were 24 male students who did not exercise regularly in grade 4-6 of a primary school in a city of China, and whose body mass index were more than $25 \mathrm{~kg} / \mathrm{m}^{2}$. They were divided into Taekwondo Poomsae training group and control group, with 12 students in each group. The training group has Taekwondo Poomsae training with a total of 12 weeks, three times a week at 4-5 p.m. After 12 weeks of Taekwondo Poomsae training, the weight, body fat rate and body mass index of the students were measured, and the results were analyzed by Statistical Product and Service Solutions (SPSS). After 12 weeks, the experimental results were as follows. After 12 weeks of training, the body weight, body fat rate and body mass index of obese students decreased significantly $(P<0.01)$. Therefore, it can be proved that Taekwondo Poomsae training has a positive effect on weight loss of obese children.
\end{abstract}

\section{Keywords: obese children, Taekwondo Poomsae, exercise intervention}

\section{INTRODUCTION}

In recent years, obesity has become an important health problem due to the improvement of China's economic level and the change of life style. According to the fourth nutritional health survey report in China, the obesity rate of 6-17-year-old children and adolescents in China tripled in the 10 years from 2002 to 2012, and the overweight rate doubled. Nowadays, children's obesity is not only a concern of the United States and other developed countries, but also a common concern of the world. According to the 2017 China children's obesity report, from 1985 to 2014, the overweight rate of school-age children over 7 years old increased from $2.1 \%$ to $12.2 \%$, while the obesity rate increased from $0.5 \%$ to $7.3 \%$, and the number of overweight and obese people increased from 6.15 million to 34.96 million [1]. Childhood and adolescence obesity is likely to be associated with adult obesity. Childhood obesity is an obstacle to maintain and improve health. It will not only lead to hypertension, diabetes, hyperlipidemia, arteriosclerosis and other cardiovascular diseases, but also a major obstacle for students in school and social life. It will have a negative impact on necessary interpersonal relationships and cause an impediment to growth and development [2].

The health problems related to obesity revealed clinically include: hypertension arteriosclerotic inflammation, chronic inflammation, induced sleep apnea asthma [3]. These are also related to metabolic syndrome, which will lead to abnormal levels of blood vessel pressure of cholesterol neutral fat insulin. In order to improve this kind of obesity, the continuous use of diet control, sports and other methods can change the state of life and achieve the purpose of energy supply and demand balance.

Taekwondo is not only the inherent martial arts of Korean Peninsula, but also a world-wide fighting sport. It cultivates personality and improves physical strength through spiritual training, which is an effective sports to promote health. Taekwondo can not only increase the physical activities, obesity management and physical strength of obese children, but also improve their mental and physical health. Luo Yirong randomly selected 40 students of the third grade of Yueyahe primary school in Hebei District of Tianjin, who basically have the same height and body shape, and divided them into two groups: the experimental group and the control group. The experimental group is the intervention group of Taekwondo exercise. The experiment lasts 16 weeks, with three classes a week, and 2 hours each class. At the end of 16 weeks, the experimental group showed significant weight loss and BMI reduction, especially, the decrease in body fat was the most obvious [4]. Unfortunately, there is no Taekwondo intervention experiment for obese children in China. However, Kwon Yoo Chan, Korean scholar, once conducted 12 weeks of Taekwondo training intervention for obese children in South Korea. The results showed that after 12 weeks of training, the body 
fat rate and total sterol of students were significantly different from those before training.

Therefore, the purpose of this study is to find out the influence of Taekwondo Poomsae training on the body composition of obese children, hoping to help prevent obesity and improve health of obese children.

\section{RESEARCH METHODS}

\section{A. Experimental method}

In this study, 24 male students in grade 4-6 of a primary school in a city of China were divided into
Taekwondo Poomsae training group and control group, with 12 students in each group. Taekwondo training group carried out 12 weeks of exercises, with three times a week and one hour training each time. The control group did not carry out Taekwondo training, only engaged in normal life and physical activities, and had no additional physical activities. The basic information of the objects is shown in "Table I".

TABLE I. BASIC INFORMATION OF STUDENTS

\begin{tabular}{|l|l|l|}
\hline \multicolumn{1}{|c|}{ Information } & \multicolumn{1}{|c|}{ Experimental group $(\mathbf{n}=\mathbf{1 2})$} & \multicolumn{1}{c|}{ Control group $(\mathbf{n}=\mathbf{1 2})$} \\
\hline Age & $11.17 \pm .72$ & $11.33 \pm 0.65$ \\
\hline Stature & $151.18 \pm 1.47$ & $149.96 \pm 2.13$ \\
\hline Weight & $58.35 \pm 1.17$ & $57.86 \pm 1.99$ \\
\hline Body fat rate & $34.57 \pm 1.00$ & $33.07 \pm 1.95$ \\
\hline $\begin{array}{l}\text { Body mass } \\
\text { index }\end{array}$ & $25.67 \pm .29$ & $25.72 \pm 0.92$ \\
\hline
\end{tabular}

1) Measuring tools and requirements: In this study, Omron HBF-514C body composition monitor and scale were used to measure the students' body weight, body mass index and body fat rate. For more accurate examination, all objects tried to wear light clothes to remove metal substances that may hinder the current flow, and limit the water intake in the time before examination.
2) Time and intensity of Taekwondo Poomsae training: Taekwondo Poomsae training in this study is based on the 2009 ACSM exercise prescription guidelines. The total training time is 12 weeks, with three times a week and one hour each time. In each training, there are 10 minutes for preparation and stretching, and 40 minutes for technical training. The training contents are shown in "Table II".

TABLE II. TAEKWONDO POOMSAE TRAINING CONTENTS

\begin{tabular}{|l|l|l|l|}
\hline \multicolumn{1}{|c|}{ Training contents } & Weeks & \multicolumn{1}{c|}{ Training contents } & \multicolumn{1}{|c|}{ Intensity (rpe) } \\
\hline Cooling down (10min) & & Stretching exercise & 9 \\
\hline & $1-4$ & Tai Ji 1 (18 Movements) & 11 (Easy) \\
\hline $\begin{array}{l}\text { Technique } \\
\text { Training (40min) }\end{array}$ & $5-8$ & Tai Ji 2 (18 Movements) & - \\
\hline & $9-12$ & Tai Ji 3 (20 Movements) & 13 (A little difficult) \\
\hline Cooling down & & Stretching exercise & 9 \\
\hline
\end{tabular}

The intensity of exercise is based on the rating scale of perceived exertion developed by Gunnar Borg, a Swedish psychologist. The exercise rating of cooling down and preparation exercise is 9 (very easy), and the exercise intensity of maintaining $50-60 \%$ of the maximum heart rate in technical training is 11 (easy) 13 (a little difficult) corresponding to the exercise scale.

\section{B. Document analysis method}

In this study, SPSS was used to analyze the experimental results and calculate the mean value and standard deviation of the two groups. In order to determine the difference between the two groups in different test time, two way repeated-measures ANOVA was selected.

\section{RESEARCH RESULTS}

In order to prove the effect of Taekwondo Poomsae training on weight loss of obese children, this study conducted 12 weeks of experimental intervention on obese children. The results of experimental intervention are shown in "Table III". 
TABLE III. ANALYSIS RESULTS OF BINARY VARIANCE

\begin{tabular}{|c|c|c|c|c|c|c|}
\hline \multirow[t]{2}{*}{ Project } & Group & Before testing & After test & \multicolumn{3}{|c|}{ Binary variance analysis } \\
\hline & & & & Group & Test period & Group $\mathrm{x}$ test time \\
\hline Weight & Experimental group & $58.35 \pm 1.71$ & $54.43 \pm 1.72$ & 3.593 & $158.595 * * *$ & $142.047 * * *$ \\
\hline$(\mathrm{kg})$ & Control group & $57.86 \pm 1.99$ & $57.76 \pm 2.06$ & & & \\
\hline Body fat rate & Experimental group & $34.57 \pm 1.00$ & $31.40 \pm 1.02$ & 0.000 & $170.207 * * *$ & $134.987 * * *$ \\
\hline$(\%)$ & Control group & $33.07 \pm .95$ & $32.88 \pm .94$ & & & \\
\hline mass & Experimental group & $25.67 \pm .29$ & $23.91 \pm .62$ & $20.209 * * *$ & $77.904 * * * *$ & 60.321 *** \\
\hline & Control group & $25.72 \pm .52$ & $25.61 \pm .66$ & & & \\
\hline
\end{tabular}

As shown in "Table III", there was no significant difference in weight between the two groups under the influence of group factors, but there was a very significant difference under the influence of time factors $(\mathrm{P}<0.01)$, and there was also a very significant difference in group and time interaction effect $(\mathrm{P}<$ $0.01)$.

There was no significant difference in body fat rate under group factors, but there was significant difference under test period factors $(\mathrm{P}<0.01)$, and there was also significant difference between group and time interaction effect $(\mathrm{P}<0.01)$.

BMI was significantly different among groups, test time, group and time $(\mathrm{P}<0.01)$.

\section{RESUlt ANALYSIS}

Among the many factors that make up body composition, body fat mass and body fat rate have an important influence on obesity, health and sports. The distribution of these factors affects the normal growth and development, so it is very important. For young children, school physical education is the foundation and the most important part of physical education, and the role of school physical education is very important. At present, there is still a phenomenon of focusing on examination subjects rather than non-examination subjects in primary and secondary schools. For the school physical education, some education departments and related principals still have the phenomenon of not being able to raise awareness. In some primary and secondary schools, the opening rate of physical education is often not satisfied. Therefore, the Third Plenary Session of the 18th Central Committee of the Communist Party of China made a clear plan for school physical education, clearly proposed the requirements of "strengthening physical education and extracurricular exercise, promoting physical and mental health of young people, and physical fitness", which will have a significant and far-reaching impact on physical education [6].

In many previous studies on obese children in growing period, Owens, a scholar, took obese children as objects and carried out aerobic exercise for four months without restriction of diet. The results showed that the body fat rate of obese children decreased by $2.2 \%$ [7]. For the effect of short-term exercise intervention, Xiao Bo once took obese children as the objects of aerobic exercise experiment intervention. The experimental group exercised three times a day, with one hour each time, for five weeks of aerobic training. Before and after the exercise, 10-20 minutes of preparatory activities were carried out respectively. There was no aerobic exercise in the control group. According to the results, the body weight, body mass index, body fat ratio and body fat content of the experimental group were significantly reduced compared with that of the control group [8].

Xie Chao, Jin Chengji and Zhang Jun used Metaanalysis to test the intervention effect of aerobic exercise on obese children in China. They conducted meta-analysis on 23 related literatures from 2005 to 2010 , and used random effect model to test the effect value and heterogeneity of the study. The results showed that the effect value of meta-analysis was $2.59,95 \%$ CI $(2.14,3.05)$, which indicated that aerobic exercise intervention had a good effect on obesity of children in China. The $\mathrm{Q}$ value of heterogeneity test index was $21.13(\mathrm{P}=0.51)$, and $\mathrm{I} 2=0 \%$. There was no heterogeneity and publication bias among the studies. The conclusion shows that aerobic exercise is an effective way for obese children to lose weight, and the effect of intervention is very obvious. Aerobic exercise lasts for more than 3 months, with 3-6 times a week, 40-90 minutes of moderate intensity exercise each time, which has the best effect on weight loss of children in China. In this study, Taekwondo Poomsae training set the exercise intensity as medium intensity exercise for 60 minutes, lasting for 12 weeks, three times a week. Under such exercise intervention, students' weight, body fat rate and body mass index also decreased. So this study is consistent with the study of Xie Chao et al.

\section{CONCLUSION}

This study takes obese children as the research objects to explore the influence of Taekwondo Poomsae training on obese children. After the experiment, the 
following conclusions can be drawn according to the research results.

First, there was no significant difference in body weight under the influence of group factors, but there was a very significant difference under the time factor $(\mathrm{P}<0.01)$, and there was also a very significant difference in the interaction effect between group and time $(\mathrm{P}<0.01)$.

Secondly, there was no significant difference in body fat rate under group factors, but there was significant difference under test period factors $(\mathrm{P}<$ $0.01)$, and there was also significant difference between group and time interaction effect $(\mathrm{P}<0.01)$.

Thirdly, there were significant differences in BMI in group, test time, group and time interaction effect $(\mathrm{P}<$ $0.01)$.

From the above results, regular Taekwondo Poomsae training can reduce the weight, body mass index and body fat rate of obese children. In the future research, in order to make obese children have fun and active sports, it is necessary to popularize a variety of sports, and increase school sports time. When students insist on physical activities, it is not only to prevent obesity, but also to live a healthy and lively school life.

\section{References}

[1] Zhang $\mathrm{Na}$, Ma Guansheng. Interpretation of Report on Childhood Obesity in China [J]. Acta Nutrimenta Sinica, 2017, 39(06): 530-534. (in Chinese)

[2] Huang Yide,Cai Lixin. Research progress in the promotion strategies of childhood obesity and health in China [J]. Maternal and Child Health Care of China, 2013, 28(18): 3039-3041. (in Chinese)

[3] Harvey Kate Christina,McDermott Helen,Coles William,Elliott Susanne,McMullan Nicola,Anandaram Archana,Ismail Sanaa,Webb Emma,Kirk Jeremy. Tackling the childhood obesity crisis: acute paediatricians are not playing their part.[J]. Pubmed,2017,102(9).

[4] Luo Yirong. Effects of Taekwondo on the body composition of primary school students [D]. Beijing Sport University, 2017. (in Chinese)

[5] Quan Yu-can, Park Sang-jia, Kim En-hee, Park Chun-ki, Zhang Zai-hee, Effects of Taekwondo Practice on Body Composition and Physical Strength and Serum of Obese Children [J]. Korean Dance Society. 12(2),239-251.

[6] Yu Jian. The dilemma of "strengthening physical education and extra-curricular exercise" in primary and secondary schools and solutions [J]. Educational exploration, 2015(03):17-19. (in Chinese)

[7] Owens, Scott, et al. "Effect of physical training on total and visceral fat in obese children $[\mathrm{J}]$. Medicine \& Science in Sports \& Exercise 31.1 (1999): 143-148.

[8] Xiao Bo. Analysis on the effects of aerobic exercise on body fat consumption of obese children [J]. Science and Technology Bulletin, 2015, 31(06): 19-21. (in Chinese)

[9] Xie Chao, Jin Chengji, Zhang Jun. The Effects of Aerobic Exercise on Treating Chinese Obese Children and Adolescents: A Meta-analysis - Based on the Component Analysis Method
[J]. Journal of Nanjing Institute of Physical Education (Social Science Edition), 2016, 30(02): 84- 90. (in Chinese) 